\title{
Optical Wavelet Signals Processing and Multiplexing
}

\author{
Gabriella Cincotti \\ Department of Applied Electronics, University Rome Tre, Via della Vasca Navale 84, 00146 Roma, Italy \\ Email:g.cincotti@uniroma3.it \\ Michela Svaluto Moreolo \\ Department of Applied Electronics, University Rome Tre, Via della Vasca Navale 84, 00146 Roma, Italy \\ Email:svaluto@uniroma3.it \\ Alessandro Neri \\ Department of Applied Electronics, University Rome Tre, Via della Vasca Navale 84, 00146 Roma, Italy \\ Email:neri@uniroma3.it
}

Received 31 March 2004; Revised 1 February 2005

\begin{abstract}
We present compact integrable architectures to perform the discrete wavelet transform (DWT) and the wavelet packet (WP) decomposition of an optical digital signal, and we show that the combined use of planar lightwave circuits (PLC) technology and multiresolution analysis (MRA) can add flexibility to current multiple access optical networks. We furnish the design guidelines to synthesize wavelet filters as two-port lattice-form planar devices, and we give some examples of optical signal denoising and compression/decompression techniques in the wavelet domain. Finally, we present a fully optical wavelet packet division multiplexing (WPDM) scheme where data signals are waveform-coded onto wavelet atom functions for transmission, and numerically evaluate its performances.
\end{abstract}

Keywords and phrases: optical communication system, wavelet transforms, wavelet packet division multiplexing, planar lightwave circuits, optical signal processing.

\section{INTRODUCTION}

Global communication networks infrastructure has been expanded thanks to recent advances in optical technology, such as transparent photonic switches and ultra-long-haul transmission systems, and the increase of multimedia data traffic is strengthening the demand for ultra-high-capacity photonic networks. The key feature of ultrafast optical networks is that the electrical conversion is avoided until data signals reach the most external edge node. Optical signal processing is not only faster than the electrical one, but it can also support a larger throughput, and current optical networks are evolving toward solutions where photonic routers are replacing electronic routers in the intermediate nodes [1].

In the present paper, we show that the use of wavelet functions in optical communications can provide an overall capacity improvement thanks to the two-dimensional processing capability of multiresolution analysis (MRA), and we present different architectures in standard planar lightwave circuits (PLC) technology that perform the wavelet analysis and multiplexing of data signals directly in the optical domain.
The wavelet transform involves joint time-frequency representation of nonstationary signals using compactly supported basis functions, and MRA has been extensively used to solve a large variety of problems in different research areas. The enormous flexibility in the choice of the wavelet allows the use of optimal wavelets for specifical applications, such as image compression, signal denoising, human vision, radar, earthquake prediction, and computer vision problems, such as range detection or motion estimation $[2,3,4]$. In optics communications, wavelets have been used for timefrequency multiplexing $[5,6]$ and ultrafast image transmission [7]. In general, wavelet signal analysis is associated with an effective computational algorithm, even faster and simpler than the fast Fourier transform (FFT) algorithm [8].

The aim of the present paper is to present a complete overview of the capabilities of wavelet signal processing and multiplexing in optical communications. In Section 2, we give a brief description of the MRA, illustrating the basic properties of the subband filtering process of both discrete wavelet transform (DWT) and the wavelet packet (WP) decomposition. In Section 3, we present the guidelines to synthesize optical wavelet filters using PLC technology, and 
describe some optical implementations for the DWT and WP decomposition. Some numerical examples for optical signal denoising and compression are given in Section 4; in particular, we show that the SNR of an optical signal can be enhanced by performing the DWT of the optical signal and thresholding the detail coefficients. In addition, we evidence the correspondence of the wavelet analysis and the optical packets compression/decompression method using delay line lattice structures [9]. Finally, in Section 5 we present an innovative full optical wavelet packets division multiplexing (WPDM) scheme and numerically test its performance.

The huge bandwidth provided by optical fibers and the capability of signal processing directly in the optical layer make an attractive combination for future multiple access networks. The feasibility of optical code division multiple access (O-CDMA) systems has been demonstrated where the spectral [10] or the temporal [11] encoding results in spreading the optical pulse in the frequency or the time domain. However, a more efficient use of the time-frequency plane has the potential of proving flexible access to a larger number of multiple end users. WPDM is an emerging technique, used in wireless communications, that transmits message signals overlapped in time and frequency domains, and recovers them with very low multiple access interference (MAI) noise, thanks to the orthogonal properties of the wavelet packet functions $[12,13,14,15]$. Data signals from different users are waveform-coded onto wavelet atom functions and transmitted in a multiple access network. Wavelet atom functions are self-orthogonal against integer translations and mutualorthogonal due to different subbands occupancy; these properties ensure a better use of time-frequency plane, with respect to standard time division multiplexing (TDM), wavelength division multiplexing (WDM), and CDMA systems [12]. In its standard form, WPDM requires $N$ waveform shapers to code each user bit sequence with a different waveform, and its optical implementation is impracticable [5]. We present a compact, full optical WPDM scheme, where the transmitter is a single encoder that multiplies $N$ optical data signals from $N$ simultaneous users. The encoder gives the equivalent sequence at the root of the WP decomposition tree, and it is followed by a single modulator that completes the waveform coding, shaping the optical pulses of the composite signal with the scaling function profile. The receiver is a reversal version of the transmitter, and the same optical devices are used for both encoding and decoding the binary sequences. In addition, we show that both the WP encoder/decoder and the waveform modulator can be fabricated using PLC technology and integrated on a single device: the WP encoder/decoder can be realized as a tree of lattice-form optical delay line filters [16], whereas the modulator as a weight/phase-programmable tapped delay line filter [17].

\section{MULTIRESOLUTION ANALYSIS}

The analysis of nonstationary signals involves a compromise between how well transitions or discontinuities are located, and how finely long-term behavior can be identified. For instance, standard Fourier transform decomposes a continuous time-signal $s(t)$ into individual frequency components, using complex sinusoidal basis functions $\exp (i \omega t)$, that are infinite in extent. As these basis functions are periodic, any short duration signal spreads over the whole basis. In contrast, the MRA decomposes a signal at different scales or resolutions, using a basis whose elements are localized in both time and frequency domains, and the representation of short duration and nonstationary signals focuses on a few components, that immediately enlighten the predominant frequencies and the time occurrence of abrupt changes.

Specifically, the continuous wavelet transform (CWT) performs correlations between the signal $s(t)$ and scaled versions of the mother wavelet $\psi(t)$ :

$$
\operatorname{CWT}_{s}(a, \tau)=\frac{1}{|a|} \int s(t) \psi^{*}\left(\frac{t-\tau}{a}\right) d t,
$$

where $a$ is a real nonzero scale parameter, and $\tau$ the translation factor. If $\psi(t)$ satisfies the admissibility condition

$$
c_{\psi}=\int \frac{|\tilde{\psi}(f)|^{2}}{|f|} d f<\infty
$$

where $\widetilde{\psi}(f)$ is the Fourier transform of the mother wavelet $\psi(t)$, the signal $s(t)$ can be reconstructed by means of the inverse wavelet transform

$$
s(t)=\frac{1}{c_{\psi}} \iint \operatorname{CWT}_{s}(a, \tau) \frac{1}{\sqrt{|a|}} \psi\left(\frac{t-\tau}{a}\right) \frac{d a d \tau}{a^{2}} .
$$

Usually $\psi(t)$ is localized both in time and frequency domains, and the CWT displays the time evolution of the frequency components of a signal. In fact, by applying the Fourier Parseval formula [18], (1) can be rewritten as

$$
\operatorname{CWT}_{s}(a, \tau)=\sqrt{|a|} \int \tilde{s}(f) \tilde{\psi}^{*}(-a f) e^{-j 2 \pi f \tau} d f,
$$

where $\tilde{s}(f)$ is the Fourier transform of the signal. Therefore, the CWT can be seen as the output from a bank of filters which are constructed by dilatations/compressions of the mother wavelet. Filters obtained by dilatations of $\psi(t)$ process the low-frequency information of the signal $s(t)$, whereas the filters related to the compressed version of $\psi(t)$ analyze the high-frequency content.

More useful in digital signal processing and multiplexing are the orthogonal wavelet series expansions, derived from the CWT when scale and translation factors are constrained to discrete values. Let $\phi(t)$ be a (smooth) scaling function such that the discrete set of functions $\left\{2^{-\ell / 2} \phi\left(2^{-\ell} t-\right.\right.$ $k \Delta \tau)(\ell, k \in Z)\}$ forms an orthonormal basis for a subspace $\mathbf{V}_{\ell} \in \mathbf{L}^{2}(\mathbf{R})$; here $\Delta \tau$ is a time interval that will coincide with the inverse of the free spectral range (FSR). Let $\psi(t)$ be an admissible mother wavelet such that (a) the discrete set 
of functions $\left\{2^{-\ell / 2} \psi\left(2^{-\ell} t-k \Delta \tau\right)(\ell, k \in Z)\right\}$ forms an orthonormal basis for a subspace $\mathbf{W}_{\ell} \in \mathrm{L}^{2}(\mathbf{R})$; (b) the subspaces $\mathbf{V}_{\ell}$ and $\mathbf{W}_{\ell}$ are mutually orthogonal, that is, $\mathbf{W}_{\ell} \perp \mathbf{V}_{\ell}$; (c) the subspace $\mathbf{V}_{\ell-1}$ can be expressed as direct sum of $\mathbf{V}_{\ell}$ and $\mathbf{W}_{\ell}$, namely

$$
\mathbf{V}_{\ell-1}=\mathbf{V}_{\ell} \oplus \mathbf{W}_{\ell}
$$

Then, a signal $s(t) \in \mathbf{V}_{0}$ is represented by a smoothed approximation at resolution $2^{M}$, obtained by combining translated versions of the basic scaling function $\phi(t)$, and $M$ details at the dyadic scales $a=2^{\ell}(\ell=1,2, \ldots, M-1)$ obtained by combining shifted and dilated versions of the mother wavelet $\psi(t)$ :

$$
\begin{aligned}
s(t)= & \sum_{k} 2^{-M / 2} c_{M}[k] \phi\left(2^{-M} t-k \Delta \tau\right) \\
& +\sum_{\ell=1}^{M} \sum_{k} 2^{-\ell / 2} d_{\ell}[k] \psi\left(2^{-\ell} t-k \Delta \tau\right) .
\end{aligned}
$$

The wavelet and the scaling function satisfy the dilation equations

$$
\begin{aligned}
& \phi(t)=\sqrt{2} \sum_{k} h[k] \phi(2 t-k \Delta \tau), \\
& \psi(t)=\sqrt{2} \sum_{k} g[k] \phi(2 t-k \Delta \tau),
\end{aligned}
$$

where $g[k]$ and $h[k]$ are the coefficients of two quadrature mirror filters (QMFs)

$$
\begin{aligned}
& H(\omega)=\frac{1}{\sqrt{2}} \sum_{k} h[k] e^{-j \omega k \Delta \tau}, \\
& G(\omega)=\frac{1}{\sqrt{2}} \sum_{k} g[k] e^{-j \omega k \Delta \tau},
\end{aligned}
$$

and $\Delta \tau$ is the inverse of their FSR $[19,20]$.

The DWT is computed by using Mallat's pyramidal algorithm [8]. Let $c_{0}[n]$ be the expansion coefficients of the signal $s(t) \in \mathbf{V}_{0}$, with respect to the orthonormal basis $\phi(t-\Delta n \tau)$ :

$$
c_{0}[n]=\langle s(t), \phi(t-n \Delta \tau)\rangle=\int s(t) \phi^{*}(t-n \Delta \tau) d t
$$

where angular brackets indicate inner product. Then the coefficients $c_{0}[n]$ can be decomposed into the scaling coefficients $c_{1}[n]$ and detail coefficients $d_{1}[n]$, via recursive discrete convolutions with the lowpass $h[n]$ and highpass $g[n]$ filters, respectively, followed by subsampling of factor 2 :

$$
\begin{aligned}
& c_{1}[n]=\sum_{k} c_{0}[k] h[2 n-k], \\
& d_{1}[n]=\sum_{k} c_{0}[k] g[2 n-k] .
\end{aligned}
$$

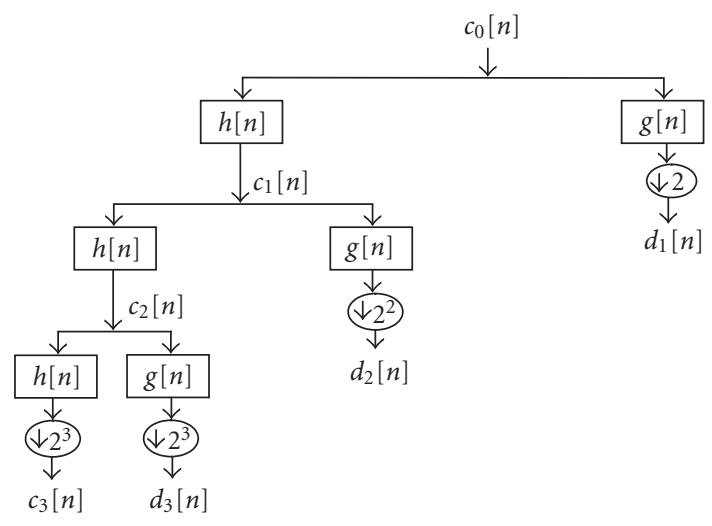

Figure 1: Pyramidal decomposition scheme for the DWT: an optical signal is driven into the device input and the optical wavelet detail and scaling coefficients are obtained at the device outputs. The output signals have to be subsampled according to their decomposition level.

The DWT decomposition halves the time resolution and doubles the frequency resolution, because the frequency band of the output signals spans only half of the frequency band of the original signal; therefore, half of the samples can be discarded by subsampling, without any information loss. On the other hand, the reconstruction process yields the input sequence by the inverse filtering and upsampling:

$$
c_{0}[n]=\sum_{k} c_{1}[k] h[2 k-n]+d_{1}[k] g[2 k-n] .
$$

The decomposition procedure can be further repeated, and, at each decomposition level $\ell$, the filtering and subsampling halves both the number of the samples and the frequency band:

$$
\begin{aligned}
& c_{\ell}[n]=\sum_{k} c_{\ell-1}[k] h[2 n-k], \\
& d_{\ell}[n]=\sum_{k} c_{\ell-1}[k] g[2 n-k] .
\end{aligned}
$$

Here, $c_{\ell}[k]$ and $d_{\ell}[k]$ are the scaling and the details coefficients, respectively, at resolution $2^{\ell}$. This approach reduces the computational load, with respect to the standard FFT, since at each decomposition level a reduced number of samples are processed, and, at the same time, improves both the time and frequency resolution. In fact, high frequencies are resolved better in the time domain and low frequencies better in the frequency domain. The pyramidal scheme for DWT is depicted in Figure 1 and we observe that only the scaling coefficients $c_{\ell}[n]$ are recursively filtered, whereas the detail coefficients $d_{\ell}[n]$ are never reanalyzed. In addition, the decimation of the wavelet coefficients is performed by subsampling according to the decomposition level, so that the number of bits in the input signal coincides with the total number of bits in all the DWT coefficients. 


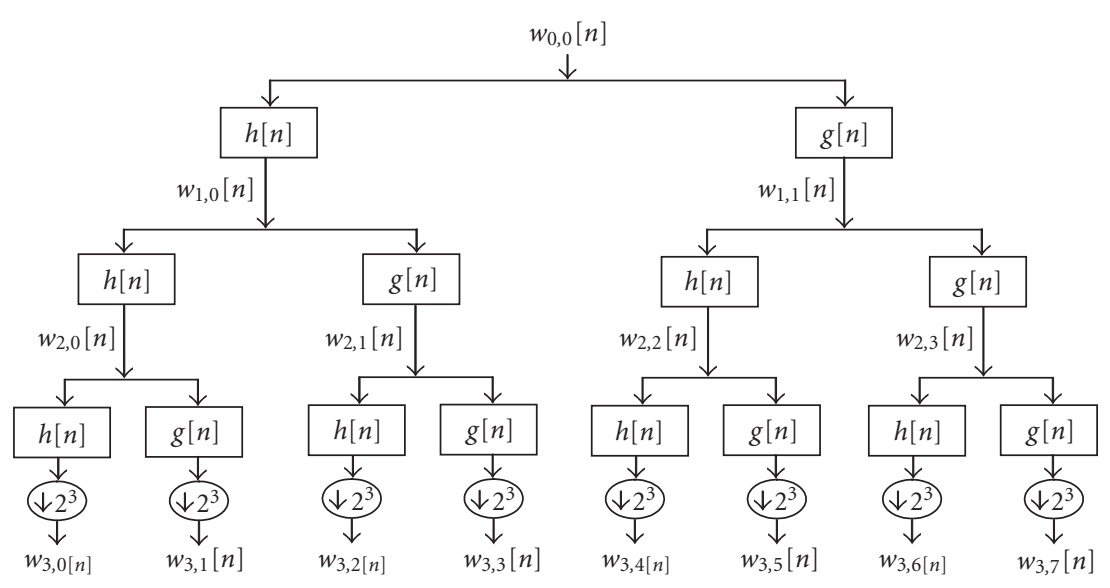

FIgure 2: Pyramidal scheme for the WP decomposition: an optical signal is driven into the device input and the optical wavelet packet coefficients are obtained at the device input. The decomposition level is $\ell=3$, and all the output signals have to be subsampled at $2^{\ell}=8$.

The WP decomposition offers a richer signal analysis, with respect to DWT, as both the scaling $c_{\ell}[n]$ and the detail coefficients $d_{\ell}[n]$ are recursively decomposed, following the same filtering and subsampling scheme [3]. The decomposition tree corresponding to a full WP analysis is illustrated in Figure 2: in this case, all the outputs have the same number of samples and span over the same frequency bandwidth, because they correspond to the same decomposition level. The WP decomposition is performed by computing the convolution of the input signal $c_{0}[n]$ with the wavelet atom functions that are a set of functions defined in the following recursive manner:

$$
\begin{aligned}
w_{\ell+1,2 m}(t) & =\sum_{k} h[k] w_{\ell, m}\left(t-2^{\ell} k \Delta \tau\right), \\
w_{\ell+1,2 m+1}(t) & =\sum_{k} g[k] w_{\ell, m}\left(t-2^{\ell} k \Delta \tau\right) .
\end{aligned}
$$

Here $\ell$ is the decomposition level, $m\left(0 \leq m \leq 2^{\ell}-1\right)$ the wavelet atom position in the tree [4]; in addition, the function $w_{0,0}(t)$ coincides with the scaling function $\phi(t)$. By exploiting the recursive structure of (13), a wavelet atom function can be expressed as

$$
w_{\ell, m}(t)=\sum_{k} f_{\ell, m}[k] \phi(t-k \Delta \tau)
$$

with $f_{\ell, m}[n]$ being the equivalent filter from the root to the $(\ell, m)$ th terminal, that can be recursively evaluated using (13). The WP atoms are self- and mutual-orthogonal functions at integer multiples of dyadic intervals [21]:

$$
\begin{array}{r}
\left\langle w_{\ell, m}\left(t-2^{\ell} n \tau\right) w_{\lambda, \mu}\left(t-2^{\lambda} k \tau\right)\right\rangle=\delta[\ell-\lambda] \delta[m-\mu] \delta[n-k], \\
\ell, \lambda \in Z, \quad 0 \leq m \leq 2^{\ell}-1, \quad 0 \leq \mu \leq 2^{\lambda}-1, \quad n, k \in Z .
\end{array}
$$

This property, that is, the waveform orthogonality is used in the WPDM to transmit multiple message signals overlapped in time and frequency domains.

\section{SYNTHESIS OF OPTICAL WAVELET FILTERS}

To implement the DWT or the WP decomposition of an optical signal, we consider the tree structures of Figures 1 and 2, where each pair $H(\omega)$ and $G(\omega)$ of QMFs is synthesized as a two-port lattice-form planar filter. If an optical digital signal, with bit rate $B$ is driven into the device input, at the device outputs we obtain the DWT or the WP decomposition, up to the $\ell$ th level. In the standard MRA, the outputs have to be subsampled at a factor $2^{\ell}$, so that the total number of the bits in the input signal coincides with the total number of bits in all the wavelet coefficient signals.

In [22], we presented the design guidelines to synthesize optical wavelet filters, following the algorithm proposed by Jinguji and Oguma [23] and using the power half-band (HB) property of wavelet filters:

$$
\begin{gathered}
|H(\omega)|^{2}+|G(\omega)|^{2}=1, \\
G(\omega)=e^{-j \omega \Delta \tau} H^{*}\left(\omega+\frac{\pi}{\Delta \tau}\right) .
\end{gathered}
$$

Jinguji and Oguma showed that an optical HB filter of length $M$ can be fabricated using a Mach-Zehnder interferometer (MZI) with a path delay difference $\Delta \tau$, and $M / 2-1$ MZIs with a path delay difference of $2 \Delta \tau$ [23]. The first MZI has an input $3 \mathrm{~dB}$ coupler and no phase shifter inserted in its arms; the remaining circuit parameters can be calculated by applying a recursive algorithm. The synthesis procedure of QMFs is quite immediate, as the circuit parameters are directly related to the filter coefficients [22]. For instance, the Daubechies wavelet filters of length $M=2$, also known as the Haar wavelet, are

$$
\left(\begin{array}{l}
H_{d B 1} \\
G_{d B 1}
\end{array}\right)=\frac{1}{\sqrt{2}}\left(\begin{array}{cc}
1 & 1 \\
1 & -1
\end{array}\right)
$$

In this case, the optical architecture for the DWT is depicted in Figure 3: each wavelet filter is synthesized as a single MZI 


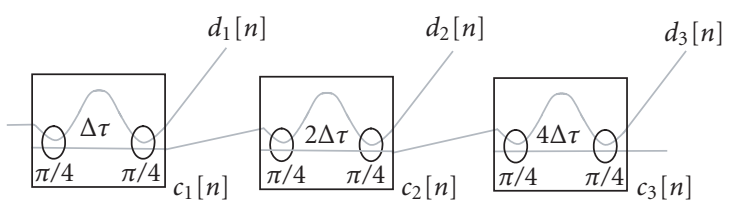

FIgURE 3: Optical architecture for the DWT with Daubechies wavelet of length $M=2$. Each optical wavelet filter is an MZI with input/output $3 \mathrm{~dB}$ couplers, and FSR that decreases from the root to the leaves in a logarithmic way.

with input/output $3 \mathrm{~dB}$ couplers; furthermore, the FSRs in the decomposition tree decrease from the root to the leaves in a logarithmic way, since at each decomposition level $\ell$, the subsampling halves the signal frequency band.

The QMFs of Daubechies wavelet of length $M=4$ are

$$
\left(\begin{array}{c}
H_{d B 2} \\
G_{d B 2}
\end{array}\right)=\frac{1}{4 \sqrt{2}}\left(\begin{array}{cccc}
1-\sqrt{3} & 3-\sqrt{3} & 3+\sqrt{3} & 1+\sqrt{3} \\
1+\sqrt{3} & -(3+\sqrt{3}) & 3-\sqrt{3} & -(1-\sqrt{3})
\end{array}\right)
$$

and the WP decomposition corresponds to the circuital scheme of Figure 4.

\section{OPTICAL WAVELET SIGNAL PROCESSING}

Wavelets are a powerful tool to denoise signal corrupted by white Gaussian noise $[24,25,26]$. The denoising scheme is based on the principle of selective wavelet reconstruction: in fact an inhomogeneous signal compacts into just a few wavelet coefficients, whereas white noise is distributed over a large number of coefficients, and, therefore, it can be reduced by thresholding the detail wavelet coefficients. The denoised signal is then obtained by inverse wavelet transforming the thresholded coefficients.

The time and frequency localization properties of the wavelet transform can also take the chromatic dispersion into account, since dispersion affects only the detail coefficients that represent highpass-filtered versions of the original signal. In this case it is necessary to perform a selective reconstruction of the wavelet coefficients that will be the subject of a next paper.

To give a numerical example of the proposed denoising method, we consider a 128-bit-long pseudorandom bit sequence (PSRS) at $B=10 \mathrm{Gbps}$ modulated by an external Mach-Zehnder modulator with $30 \mathrm{~dB}$ extinction ratio; the signal SNR ratio is $24 \mathrm{~dB}$. The device of Figure 3 performs the DWT of the optical signal at $\lambda=1550 \mathrm{~nm}$, composed of Gaussian pulses of $\delta t=20$ ps width; the FSR is chosen equal to $16 / \delta t$. At the device outputs we obtain the scaling coefficients $c_{3}[n]$ at the third level of decomposition $(\ell=3)$, and all the detail coefficients $d_{\ell}[n](\ell=1,2,3)$, that are plotted in Figure 5. It is evident that all the detail coefficients are the highpass-filtered version of the signal, and they can be suppressed to eliminate the noise; therefore, the denoised signal is reconstructed using only the scaling coefficients $c_{3}[n]$. The eye diagram of the original and the denoised signals are reported in Figure 6 and we observe a significative improve- ment in the eye opening. We also remark that the efficiency of the denoising method depends on the wavelet choice, and the threshold selection rules. In the previous example, we consider a very simple case, where all the detail coefficients have been completely eliminated, but better performances can be achieved with optimal thresholding methods.

To quantify the SNR improvement, in Figure 7 we plot both the SNR and the BER of the denoised signal, as functions of the SNR of the input signal, and we observe that the SNR is increased by more than $10 \mathrm{~dB}$.

The device of Figure 3 can be also used to compress/decompress optical data in ultrafast packet-switched networks. In fact the delay lattice scheme proposed by Toliver et al. in [9] is an incomplete DWT scheme that evaluates only the scaling coefficients $c_{\ell}[n]$. In this case, the unit delay $\Delta \tau$ equates the difference between the pulse periods in the uncompressed and compressed signals. It is evident that a standard DWT or a full WP decomposition allows a larger variety of compression/decompression methods.

\section{OPTICAL WAVELET PACKET DIVISION MULTIPLEXING}

The success and widespread use of code division multiple access (CDMA) in the wireless domain has renewed interest in exploring its use in the optical domain, which, however, presents a different set of challenges [27]. In an optical CDMA (O-CDMA) network, the information sequences are codified in time, using temporal codes, or in frequency domain with standard diffraction-based spread-spectrum techniques. Recently, hybrid techniques have been proposed to manage burst changes of data traffic or increasing requests of new data services [28]. O-CDMA can support high-capacity services, broadband signals processing, and multiplexing of a large number of users; in addition, many approaches have been proposed to avoid the optical-electrical-optical conversion, that severely limits the transmission system performances.

We present an all-optical WPDM system that makes a very efficient use of time-frequency plane. The digital sequences from each user are encoded by a set of orthogonal waveforms [12]: the orthogonal properties of the wavelet atoms and their overlapping nature in time and frequency yield an overall system capacity improvement [5].

The TDM sequence $\sigma_{\ell, m}[n]$ from the $m$ th user at bit rate $B=1 / T$ is waveform-coded by the wavelet atom function $w_{\ell, m}(t)$ :

$$
s_{\ell, m}(t)=\sum_{n} \sigma_{\ell, m}[n] w_{\ell, m}\left(t-2^{\ell} n \Delta \tau\right)
$$

and summed together with the waveform-coded signals from the other $2^{\ell}-1$ users:

$$
s_{c}(t)=\sum_{m=0}^{2^{\ell}-1} \sum_{n} \sigma_{\ell, m}[n] w_{\ell, m}\left(t-2^{\ell} n \Delta \tau\right)
$$




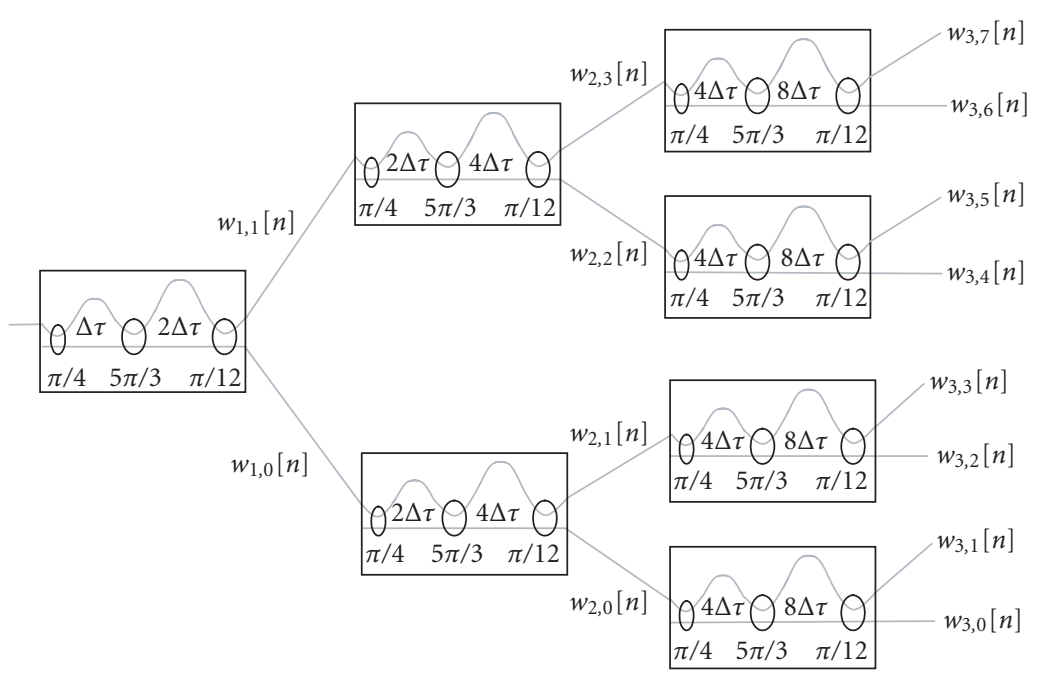

FIgure 4: Optical architecture for the WP decomposition with Daubechies wavelet filter of length $M=4$. Each optical wavelet filter is a chain of two MZIs, with FSR that decreases from the root to the leaves in a logarithmic way.

The processing gain of the WPDM system is equivalent to the O-CDMA parameter and is expressed as the ratio of the time duration of the waveform and the input signal width $\delta t$ [29]:

$$
F=\frac{2^{\ell} \Delta \tau}{\delta t}
$$

For a correct waveform shaping, it is necessary that the input bit duration $\delta t$ equates the inverse of the FSR, that is, $\delta t=\Delta \tau$ : in this way, each optical pulse is transformed into the corresponding wavelet atom function at the device output. Therefore, the processing gain $F=2^{\ell}$ equates the number of simultaneous users.

A standard WPDM transmission system consists of a bank of waveform modulators, one for each user, and its optical implementation is really complex [5]. A more compact WPDM architecture, with a single WP encoder and only one waveform modulator, can be designed by "reversing" the expression of $(20)$ for the composite signal $s_{c}(t)$. In fact, if we substitute (14) into (20), we obtain

$$
\begin{aligned}
s_{c}(t) & =\sum_{m=0}^{2^{\ell}-1} \sum_{n} \sigma_{\ell, m}[n] \sum_{k} f_{\ell, m}[k] \phi\left[t-\left(2^{\ell} n+k\right) \Delta \tau\right] \\
& =\sum_{i} \sigma[i] \phi(t-i \Delta \tau),
\end{aligned}
$$

where

$$
\sigma[i]=\sum_{m=0}^{2^{\ell}-1} \sum_{n} f_{\ell, m}\left[i-2^{\ell} n\right] \sigma_{\ell, m}[n]
$$

is the equivalent sequence from all the users obtained at the root of the WP tree [12]. From an inspection of this equation, it is evident that the composite signal $s_{c}(t)$ can be generated by first encoding all the input sequence $\sigma_{\ell, m}[n]$ from each user with a WP encoder, thus obtaining $\sigma[i]$, and then waveform shaping all the composite bits by the scaling function $\phi(t)$ [6]. This WPDM architecture is schematically illustrated in Figure 8: the WPDM transmission system is implemented by the WP encoder of Figure 2, followed by a single modulator that shapes the multiplexed optical pulses $\sigma[i]$ with the scaling function profile $\phi(t)$. The receiver is a time reversal version of the transmitter, and the WP decoder follows the pulse shaper; therefore, the same device can be used for both encoding and decoding the binary sequences.

The time gating opens a time window to extract the autocorrelation peak and it is somewhat equivalent to narrowbandpass filtering in the wireless CDMA. The use of a time gating before detection significantly relaxes the requirements for the detector bandwidth to the bit rate of $2^{\ell} \Delta t$. The synchronization from the time gating can be derived from the recovered clock.

The optical architecture for a WP encoder/decoder is identical to an optical WP decomposition scheme, so that the scheme of Figure 4 can be thought of as optical E/D for the Daubechies wavelets of length $M=4$.

In a WPDM system, each bit from each user is waveformcoded by wavelet atom functions, and all the data streams from the users are transmitted simultaneously. On the other hand, in a code-based O-CDMA system, a different code is assigned to each user; therefore, if we remove the pulse shapers in the transmission scheme of Figure 8, we obtain a standard O-CDMA system, where the input data signals are coded by optical orthogonal codes (OOCs) sequences [30]. In fact, the device of Figure 4 is a full optical E/D that codes/decodes binary sequences from $2^{\ell}$ users simultaneously [31,32]; of course, for an O-CDMA system, it is $\delta t<\Delta \tau$. 

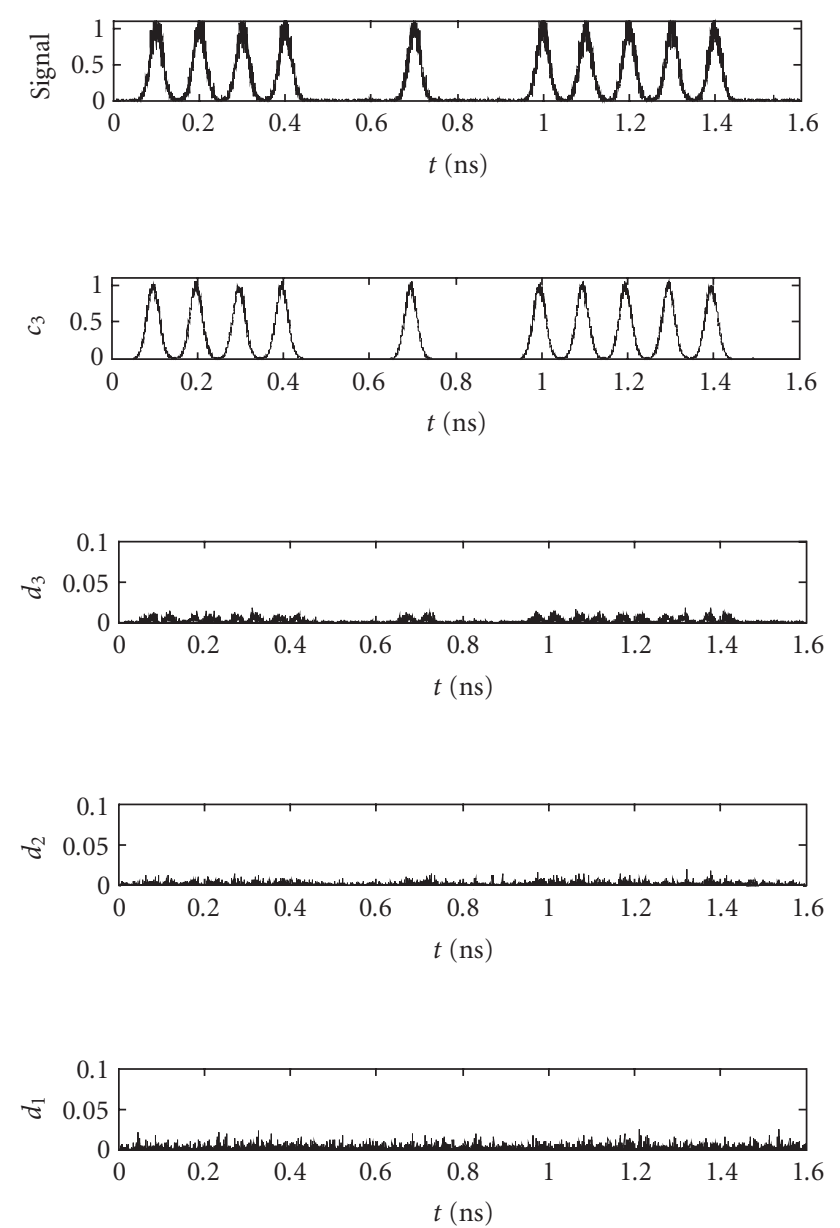

Figure 5: Input optical signal at $B=10 \mathrm{Gbps}, \lambda=1550 \mathrm{~nm}$, and $\mathrm{SNR}=24 \mathrm{~dB}$; the Gaussian optical pulses have 20 ps width. Scaling coefficients $c_{3}$ that coincide with the denoised signal and thresholded detail coefficients at levels $1,2,3$ are obtained. The DWT is performed with the device of Figure 3, with no subsampling.

To complete the waveform coding in a WPDM system, an optical pulse shaper is necessary. We consider two different devices; the first one is a diffractive pulse shaper, identical to an O-CDMA spread-spectrum encoder, composed of two diffraction gratings and an amplitude/phase filter [33]. The incoming pulses are spatially decomposed in their spectral components by the first grating, and an amplitude/phase mask gives the scaling function spectral profile to the radiation; the second grating reassembles the waveform-coded pulses. Otherwise, an optical pulse shaper can be fabricated as a weight/phase-programmable optical-tapped delay line filter [17]. In this case, both the WP E/D and the waveform shaper can be integrated on a single silica substrate.

To evaluate the detection capabilities of a full optical WPDM system, we generate $N$ independent data signals at $B=10$ Gbps modulated by $2^{7}-1$ word length PRBSs, using

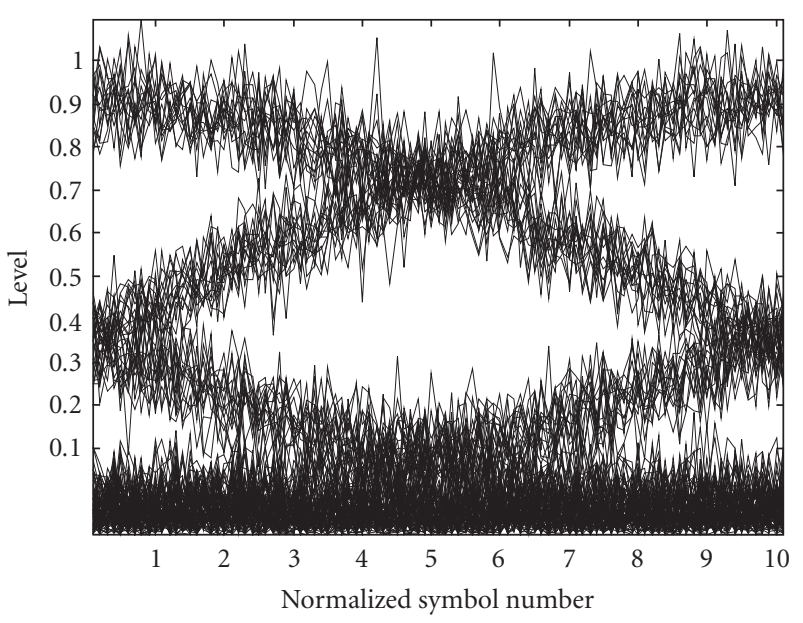

(a)

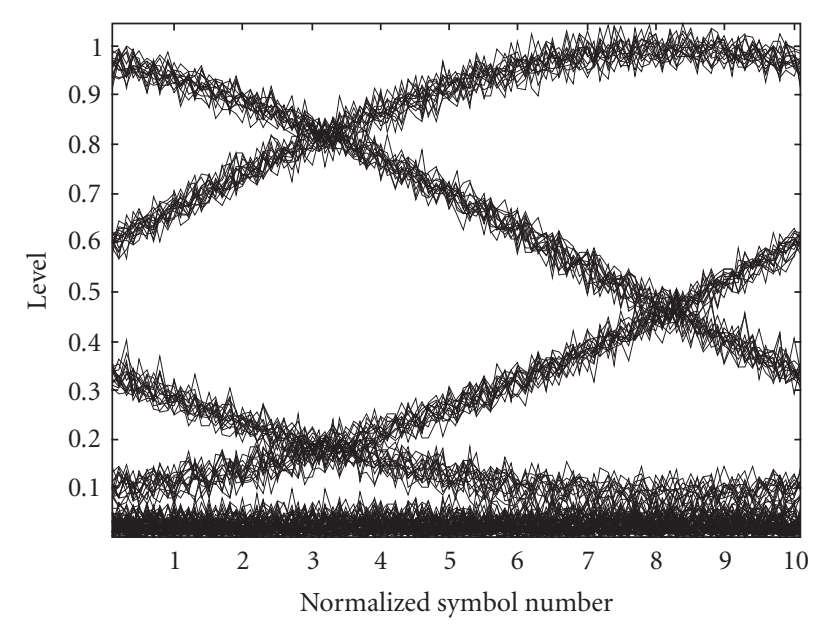

(b)

FIGURE 6: Eye pattern diagrams of the input signal and the denoised signal of Figure 5.

external Mach-Zehnder modulators with $30 \mathrm{~dB}$ extinction ratio. Each user bit sequence is forwarded to the $N$ inputs of the encoder of Figure 4 and the encoder output is waveformmodulated by the pulse shaper corresponding to the scaling wavelet of the Daubechies filters of length $M=4$, as illustrated in Figure 8. Figure 9 shows the signal-to-MAI ratio as a function of the number of the simultaneous users $N$, that is evaluated as the ratio between the signal corresponding to a " 1 " from the $i$ th user and the signal detected at the same output when the $i$ th user is transmitting a " 0 " and all the other $N-1$ users are transmitting a "1."

We finally observe that the system performances can be enhanced by a suitable choice of the wavelet decomposition [12]. 


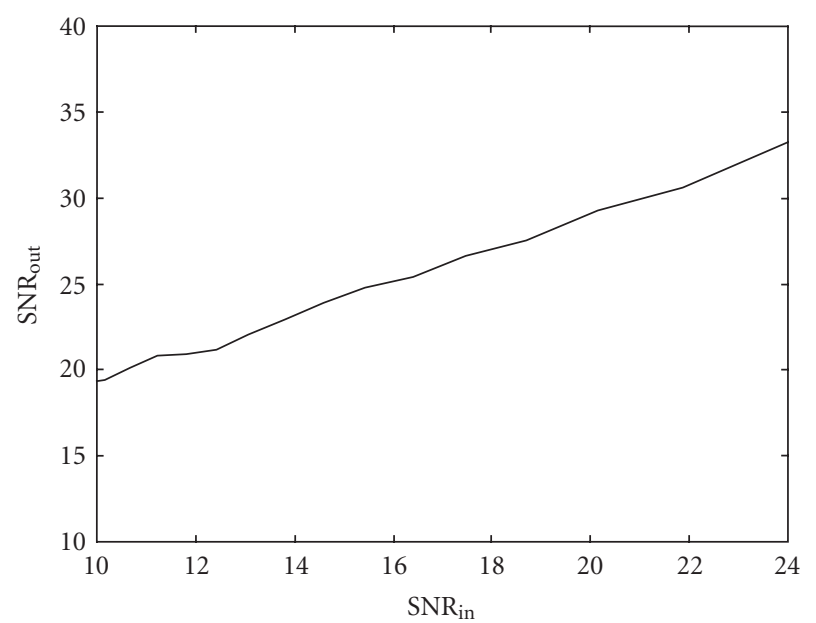

(a)

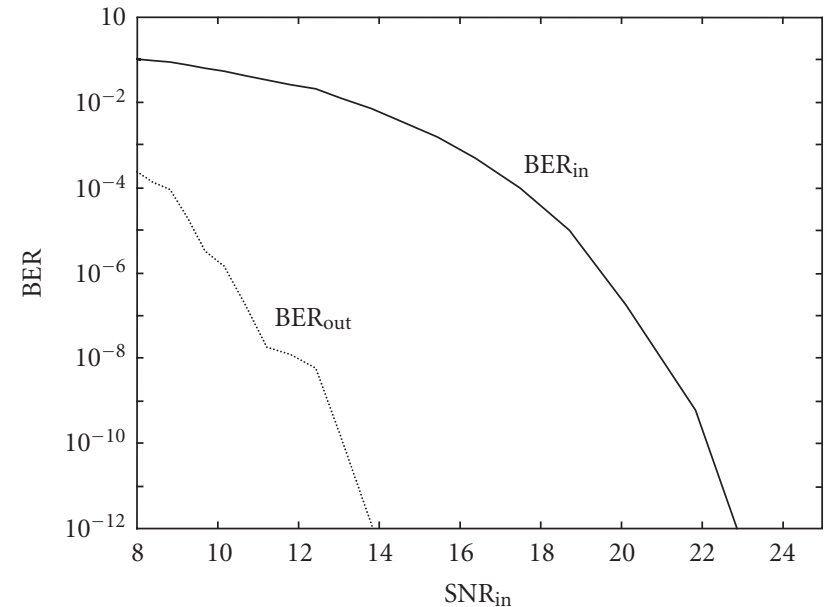

(b)

FIGURE 7: (a) SNR of the denoised signal (that coincides with the scaling coefficients $c_{3}$ from the device of Figure 3 ) versus the SNR of the input optical signal. (b) Bit error rate of the input signal (solid line) and the denoised signal (dotted line) versus the SNR of the input signal.
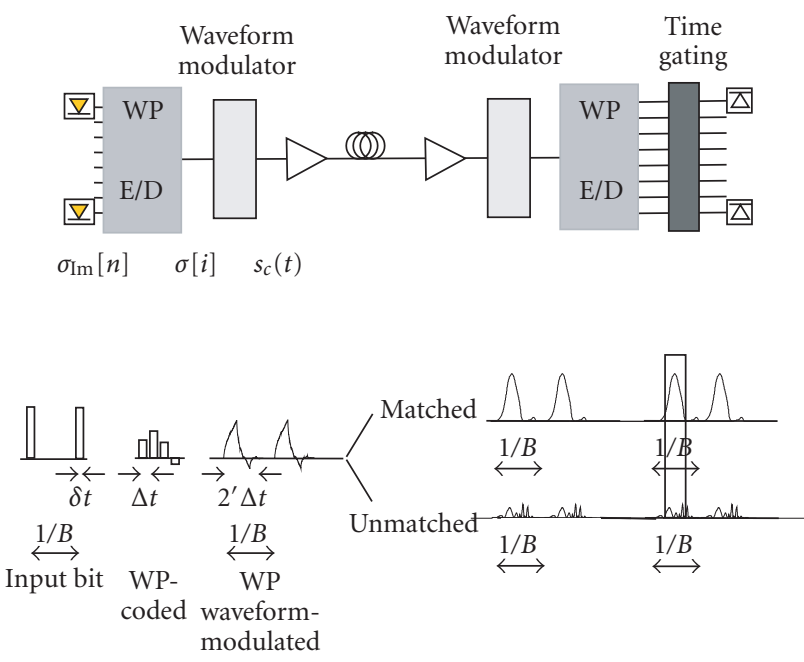

Figure 8: Schematic of an optical WPDM system: the data sequences from $N$ users are WP-encoded by a single optical device; the composite signal is then waveform-modulated by a pulse shaper. The receiver is a reverse version of the transmitter.

\section{CONCLUSION}

We present a complete overview of the wavelet signal processing and multiplexing in the optical domain, using passive PLC devices.

We describe the PLC architectures to perform the DWT and the WP decomposition of an optical digital signal, and furnish the design guidelines to synthesize a wavelet filter as a two-port lattice-form planar device. We demonstrate that within the MRA it is possible to both denoise and

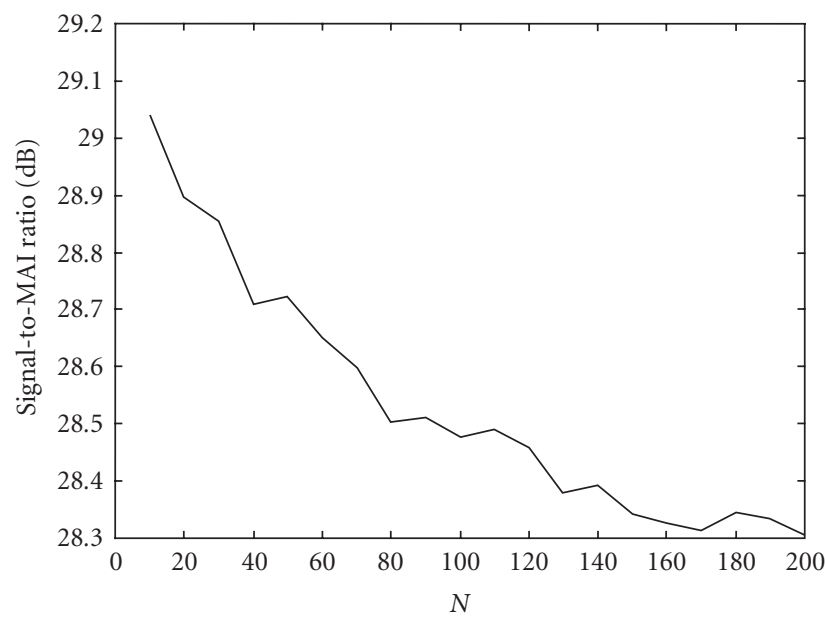

FIGURE 9: Signal-to-multiple-access-interference ratio (dB) versus the number $N$ of simultaneous users.

compress/decompress data streams directly in the optical domain; in addition we evidence the large flexibility of the proposed approaches, thanks to the possibility to choose the optimal wavelet.

Furthermore, we present an innovative full optical WPDM scheme that transmits multiple signals by waveform coding, yielding an overall capacity improvement and a more efficient use of the common shared resources, with respect to standard TDM and WDM [12]. We show that the encoded sequences are recovered with very low MAI noise, since multiple signals transmitted, overlapped in both time and frequency domains. 
The WP encoder/decoder and the pulse shaper are lowloss compact devices that can be fabricated using standard PLC technology and integrated together on a single common mechanically rigid substrate, avoiding undesirable variations of optical path lengths over time, temperature, and exposure to mechanical vibrations. The possibility of growing or pruning the wavelet decomposition tree allows to adapt the multiple access system to different traffic requirements, adding or dropping data streams.

The optical signal processing fully exploits the fibre bandwidth, and it is suitable for broadband multiple access networks. The proposed devices can be employed in ultrafast and highly-robust multiple access networks and their low cost, compactness, and fabrication simplicity, make them attractive for an ever-increasing number of different applications.

\section{REFERENCES}

[1] K.-I. Kitayama and N. Wada, "Photonic IP routing," IEEE Photon. Technol. Lett., vol. 11, no. 12, pp. 1689-1691, 1999.

[2] E. A. Rosenfeld, Multiresolution Techniques in Computer Vision, Springer-Verlag, New York, NY, USA, 1984.

[3] J. J. Benedetto and M. W. Frazier, Wavelets: Mathematics and Applications, CRC Press, Boca Raton, Fla, USA, 1994.

[4] A. K. Louis and P. Maaß, Wavelets: Theory and Applications, John Wiley \& Sons, New York, NY, USA, 1997.

[5] T. Olson, D. Healy, and U. Österberg, "Wavelets in optical communications," IEEE Computing in Science \& Engineering, vol. 1 , no. 1, pp. 51-57, 1999.

[6] G. Cincotti, M. S. Moreolo, and A. Neri, "All optical multiplexing scheme for multiple access networks based on wavelet packets filter banks," in Proc. SPIE International Symposium Integrated Photonics Europe, Strasbourg, France, 2004.

[7] T. Konishi and Y. Ichioka, "Ultrafast image transmission by optical time-to-two-dimensional-space-to-time-to-twodimensional-space conversion," Journal of the Optical Society of America $\{A\}$, vol. 16, no. 5, pp. 1076-1088, 1999.

[8] S. G. Mallat, "A theory for multiresolution signal decomposition: the wavelet representation," IEEE Trans. Pattern Anal. Machine Intell., vol. 11, no. 7, pp. 674-693, 1989.

[9] P. Toliver, K.-L. Deng, I. Glesk, and P. R. Prucnal, "Simultaneous optical compression and decompression of $100-\mathrm{Gb} / \mathrm{s}$ OTDM packets using a single bidirectional optical delay line lattice," IEEE Photon. Technol. Lett., vol. 11, no. 9, pp. 1183 $1185,1999$.

[10] R. P. Scott, et al., "Demonstration of an error-free 4 X 10-Gb/s multi-user SPEC O-CDMA network test bed," IEEE Photon. Technol. Lett., vol. 16, pp. 2186-2188, 2004.

[11] K.-I. Kitayama, "Code division multiplexing lightwave networks based upon optical code conversion," IEEE J. Select. Areas Commun., vol. 16, no. 7, pp. 1309-1319, 1998.

[12] K. M. Wong, J. Wu, T. N. Davidson, and Q. Jin, "Wavelet packet division multiplexing and wavelet packet design under timing error effects," IEEE Trans. Signal Processing, vol. 45, no. 12 , pp. 2877-2890, 1997.

[13] A. R. Lindsey, "Wavelet packet modulation for orthogonally multiplexed communication," IEEE Trans. Signal Processing, vol. 45, no. 5, pp. 1336-1339, 1997.

[14] K. M. Wong, J. Wu, T. N. Davidson, Q. Jin, and P.-C. Ching, "Performance of wavelet packet-division multiplexing in impulsive and Gaussian noise," IEEE Trans. Commun., vol. 48, no. 7, pp. 1083-1086, 2000.
[15] R. E. Learned, H. Krim, B. Claus, A. S. Willsky, and W. C. Karl, "Wavelet-packet-based multiple access communication," in Wavelet Applications in Signal and Image Processing II, vol. 2303 of Proceedings of SPIE, pp. 246-259, San Diego, Calif, USA, October 1994.

[16] G. Cincotti, "Fiber wavelet filters," IEEE J. Quantum Electron., vol. 38, no. 10, pp. 1420-1427, 2002.

[17] K.-I. Kitayama, S. Osawa, N. Wada, and W. Chujo, "Optical pulse train synthesis of arbitrary waveform using weight/phase-programmable 32-tapped delay line waveguide filter," in Optical Fiber Communication Conference and Exhibit (OFC '01), vol. 3, pp. WY3/1-WY3/3, Anaheim, Calif, USA, March 2001.

[18] R. N. Bracewell, The Fourier Transform and Its Applications, McGraw-Hill, New York, NY, USA, 1986.

[19] M. Vetterli and C. Herley, "Wavelets and filter banks: theory and design," IEEE Trans. Signal Processing, vol. 40, no. 9, pp. 2207-2232, 1992.

[20] C. Herley and M. Vetterli, "Orthogonal time-varying filter banks and wavelet packets," IEEE Trans. Signal Processing, vol. 42, no. 10, pp. 2650-2663, 1994.

[21] B. S. Krongold, K. Ramchandran, and D. L. Jones, "Frequency-shift-invariant orthonormal wavelet packet representations," IEEE Trans. Signal Processing, vol. 47, no. 9, pp. 2579-2582, 1999.

[22] G. Cincotti, M. S. Moreolo, and A. Neri, "Synthesis of optical wavelet filters," IEEE Photon. Technol. Lett., vol. 16, no. 7, pp. 1679-1681, 2004.

[23] K. Jinguji and M. Oguma, "Optical half-band filters," J. Lightwave Technol., vol. 18, no. 2, pp. 252-259, 2000.

[24] D. L. Donoho and I. M. Johnstone, "Ideal spatial adaptation by wavelet shrinkage," Biometrika, vol. 81, no. 3, pp. 425-455, 1994.

[25] L. M. Johnstone and B. W. Silverman, "Wavelet threshold estimators for data with correlated noise," Journal of the Royal Statistical Society: Series B, vol. 59, pp. 319-351, 1997.

[26] M. Lang, H. Guo, J. E. Odegard, C. S. Burrus, and R. O. Wells Jr., "Noise reduction using undecimated discrete wavelet transform," IEEE Signal Processing Lett., vol. 3, no. 1, pp. 1012, 1996.

[27] J. A. Salehi, "Code division multiple-access techniques in optical fiber networks. I. Fundamental principles," IEEE Trans. Commun., vol. 37, no. 8, pp. 824-833, 1989.

[28] T. Pfeiffer, J. Kissing, J.-P. Elbers, et al., "Coarse WDM/CDM/TDM concept for optical packet transmission in metropolitan and access networks supporting 400 channels at $2.5 \mathrm{~Gb} / \mathrm{s}$ peak rate," J. Lightwave Technol., vol. 18, no. 12, pp. 1928-1938, 2000.

[29] K.-I. Kitayama, N. Wada, and H. Sotobayashi, "Architectural considerations for photonic IP router based upon optical code correlation," J. Lightwave Technol., vol. 18, no. 12, pp. 1834$1844,2000$.

[30] T. Dennis and J. F. Young, "Optical implementation of bipolar codes," IEEE J. Quantum Electron., vol. 35, no. 3, pp. 287-291, 1999.

[31] G. Cincotti, "Full optical encoders/decoders for photonic IP routers," J. Lightwave Technol., vol. 22, no. 2, pp. 337-342, 2004.

[32] PCT patent Application No. PCT/IT03/000879 filed on December 30th 2003 by University Roma TRE, inventor Gabriella Cincotti.

[33] J. A. Salehi, A. M. Weiner, and J. P. Heritage, "Coherent ultrashort light pulse code-division multiple access communication systems," J. Lightwave Technol., vol. 8, no. 3, pp. 478-491, 1990. 
Gabriella Cincotti was born in Naples, Italy, in 1966. She received the Laurea (M.S.) degree (cum laude) in electronic engineering from University of Rome "La Sapienza" in April 1992. She was a project engineer at the Microwave Laboratory, Alenia, Aeritalia \& Selenia S.p.A., Rome, from 1992 to 1994, and joined the Department of Applied Electronics, University Rome Tre, as an Assistant Professor, in October 1994. Her ongoing research areas include passive optical devices, as optical filters, wavelength demultiplexing, encoders/decoders, and polarizing devices. Her research results have been recorded in over 50 refereed papers and summarized in nearly 20 conference and symposium presentations. She is a Member of the IEEE Lasers and Electro-Optics Society (LEOS), the Consorzio Nazionale Interuniversitario per le Telecomunicazioni (CNIT), and the National Institute for the Physics of the Matter (INFM), University Rome Tre, Rome, Italy.

Michela Svaluto Moreolo was born in Rome, Italy, in 1977. She received the Laurea (M.S.) degree (cum laude) in electronic engineering from University Rome Tre in May 2003. She is currently a Ph.D. student in telecommunication engineering at the University Rome Tre, Rome, Italy, since November 2003. Her research interests are in the field of passive optical devices, such as planar lightwave circuits and photonic bandgap structures. Her studies are directed towards all-optical signal processing in WDM systems and all-optical beamforming in radio-over-fiber systems. Her research in nanophotonics involves also quantum dot lasers/LEDs. She is a Student Member of the IEEE Lasers and Electro-Optics Society (LEOS).

Alessandro Neri was born in Viterbo in 1954. In 1977 he received the Doctoral degree in electronic engineering from the University of Rome "La Sapienza." In 1978 he joined the Research and Development Department, Contraves Italiana S.p.A., where he gained a specific expertise in the field of radar signal processing and in applied detection and estimation theory, thus becoming the Chief of the Advanced Systems Group. In 1987 he joined the INFOCOM Department, University of Rome "La Sapienza," as an Associate Professor in signal and information theory. In 1992 he joined the Department of Applied Electronics, University Rome Tre, as an Associate Professor. He became a Full Professor in telecommunications in September 2001. Since 1992, he has been responsible for the coordination and management of research and teaching activities in the telecommunication fields at the University Rome Tre. His research activity has mainly been focused on information theory, signal theory, and signal and image processing, and their applications to both telecommunications systems and remote sensing. His current research is focused on 3rd- and 4th-generation cellular systems and multimedia communications. 